\title{
Correction to: Review of the waspfish genus Neocentropogon (Tetrarogidae), with a key to genera in the family
}

\author{
Sirikanya Chungthanawong ${ }^{1,2}\left(\mathbb{D} \cdot\right.$ Hiroyuki Motomura $^{3}$
}

Published online: 15 November 2021

(c) The Ichthyological Society of Japan 2021

\section{Correction to: Ichthyol Res (2021) 68:404-425 https://doi.org/10.1007/s10228-020-00796-w}

A part of comparative specimens listed under Material examined for key to genera was missing from the original article. The missing part is as follows:

Paracentropogon longispinis (Cuvier in Cuvier and Valenciennes 1829): 9 specimens (24.6-70.5 mm SL) - FRLM 15745, 25.3 mm SL, Indonesia; FRLM 20344, 24.6 mm SL, Indonesia; FRLM 35715, 70.5 mm SL, Indonesia; FRLM 35716, $69.3 \mathrm{~mm}$ SL, Indonesia; FRLM 35730, $65.1 \mathrm{~mm}$ SL, Indonesia; FRLM 35731, 64.4 mm SL, Indonesia; FRLM 50309, $58.4 \mathrm{~mm}$ SL, Indonesia; KAUM-I. 80615, $52.6 \mathrm{~mm}$ SL, Philippines. KAUM-I. 132445, 68.0 mm SL, Singapore. Paracentropogon vespa Ogilby 1910: 6 specimens (30.4-73.2 mm SL) - AMS IA. 4236, holotype of Paracentropogon vespa livingstonei Whitley 1933, $59.0 \mathrm{~mm} \mathrm{SL}$, Australia; AMS E. 2932, holotype of P. vespa, $66.9 \mathrm{~mm}$ SL, Australia; QM I. 35818, 30.4 mm SL, Australia; QM I. 35951, 73.2 mm SL, Australia; QM I. 37374, 2, 47.0$52.7 \mathrm{~mm}$ SL, Australia. Paracentropogon rubripinnis (Temminck and Schlegel 1843): 8 specimens (40.7-72.7 mm SL)

The original article can be found online at https://doi.org/10.1007/ s10228-020-00796-w.

Sirikanya Chungthanawong

Sirikanya.c@nsm.or.th

Hiroyuki Motomura

motomura@kaum.kagoshima-u.ac.jp

1 The United Graduate School of Agricultural Sciences, Kagoshima University, 1-21-24 Korimoto, Kagoshima 890-0065, Japan

2 Present Address: Natural History Museum, National Science Museum, 39, Moo 3, Khlong 5, Khlong Luang 12120, Pathumthani, Thailand

3 The Kagoshima University Museum, 1-21-30 Korimoto, Kagoshima 890-0065, Japan
- KAUM-I. 05963, 43.2 mm SL, Japan; KAUM-I. 21120, 72.7 mm SL, Japan; KAUM-I. 21134, 57.0 mm SL, Japan; KAUM-I. 22773, 48.8 mm SL, Japan; KAUM-I. 99947, 50.2 mm SL, Japan; KAUM-I. 115740, 40.7 mm SL, Japan; KAUM-I.131459, 47.2 mm SL, Japan; KAUM-I.151038, $54.4 \mathrm{~mm}$ SL, Japan. Pseudovespicula cypho (Fowler 1938): 4 specimens (32.0-46.9 mm SL) — USNM 98902, holotype of Prosopodasys cypho, $32.0 \mathrm{~mm}$ SL, Philippines; CSIRO A 160, 46.9 mm SL, Papua New Guinea; USNM 136396, 2 , 38.3-43.3 mm SL, Philippines. Pseudovespicula dracaena (Cuvier in Cuvier and Valenciennes 1829): 12 specimens (33.2-49.0 mm SL) - MNHN 6522, 2 syntypes of Apistus belengerii Cuvier in Cuvier and Valenciennes 1829, 46.8-47.5 mm SL, India; SMF 389, syntype of Apistus dracaena, $45.4 \mathrm{~mm}$ SL, Bahrain; ZMB 807, syntype of $A$. dracaena, $38.3 \mathrm{~mm}$ SL, locality unknown; BPBM 21185, 2, 37.8-40.4 mm SL, Bahrain; MNHN 5743, 2, 39.6-41.9 mm SL, India; MNHN 7306, 2, 33.2-35.1 mm SL, India; ZSI 1738, 2, 44.1-49.0 mm SL, India. Richardsonichthys leucogaster (Richardson 1848): 11 specimens (17.1-37.2 mm SL) - AMS I. 16352-001, 42.3 mm SL, Australia; AMS I. 20751-034, 2, 34.0-55.6 mm SL, Australia; AMS I. 22832013, 2, 39.6-41.2 mm SL, Australia; BPBM 33835, 2, 17.1-37.2 mm SL, New Caledonia; QM I. 34377, 43.6 mm SL, Australia; QM I. 34939, 39.4 mm SL, Australia; QM I. 35086, 59.3 mm SL, Australia; QM I. 36069, $41.0 \mathrm{~mm}$ SL. Australia. Snyderina guentheri (Boulenger 1889): 22 specimens (46.9-175.3 mm SL) - BMNH 1888.12.29.145, holotype of Tetraroge guentheri, $164.2 \mathrm{~mm} \mathrm{SL}$, Oman; BMNH 1901.1.30.18, 96.8 mm SL, Oman; BMNH 1939.524.15561559, 4, 46.9-137.8 mm SL, Somalia; BMNH 1992.1.9.1, $142.0 \mathrm{~mm}$ SL, Indian Ocean; CAS 14651, $54.6 \mathrm{~mm} \mathrm{SL}$, India; CAS 36688, $113.7 \mathrm{~mm}$ SL, India; CAS 48621, 3, 80.0-106.0 mm SL, Oman; CAS 54600, 82.3 mm SL, Indonesia; CAS 54601, $79.1 \mathrm{~mm}$ SL, Indonesia; SAIAB 48838, $175.3 \mathrm{~mm}$ SL, South Africa; SAIAB 189168, $136.4 \mathrm{~mm}$ SL, South Africa; SAIAB 189563, 92.4 mm SL, Madagascar; SAIAB 189825, 58.6 mm SL, Madagascar; ZSI F 7181/2, 
4, 118.5-152.9 mm SL, India. Snyderina yamanokami Jordan and Starks 1901: 9 specimens (92.6-162.5 mm SL) CAS-SU 6433, holotype of S. yamanokami, $162.5 \mathrm{~mm}$ SL, Japan; KAUM-I. 17754, 92.6 mm SL, Taiwan; KAUM-I. 17770, 159.2 mm SL, Taiwan; KAUM-I. 17771, 130.8 mm SL, Taiwan; KAUM-I. 17772, 144.7 mm SL, Taiwan; KAUM-I. 17773, 134.1 mm SL, Taiwan; KAUM-I. 17774, 134.5 mm SL, Taiwan; KAUM-I. 20433, 153.9 mm SL, Taiwan; KAUM-I. 64285, 159.0 mm SL, Japan. Snyderina sp.: 6 specimens (29.6-53.5 mm SL) - MNHN 2005-0744, 6 of 7, 29.6-53.5 mm SL, Philippines. Tetraroge barbata (Cuvier in Cuvier and Valenciennes 1829): 2 specimens (68.5-70.7 mm SL) - AMS B. 8266, 68.5 mm SL, Malaysia; AMS I. 17535-003, 70.7 mm SL, Papua New Guinea. Tetraroge nigra (Cuvier in Cuvier and Valenciennes 1829): 13 specimens (24.7-100.5 mm SL) - MNHN 6638, 2 syntypes of Apistus niger, 53.6-53.8, India; AMS I. 15346001, 41.3 mm SL, Papua New Guinea; KAUM-I. 52299, 96.9 mm SL, Philippines; NSMT-P 80854, 100.5 mm SL, Japan; NSMT-P 80855, 96.5 mm SL, Japan; QM I. 40707, 87.8 mm SL, Papua New Guinea; URM-P 31819, 6 of 12, 24.7-29.4 mm SL, Fiji. Trichosomus trachinoides (Cuvier in Cuvier and Valenciennes 1829): 31 specimens (21.2$75.8 \mathrm{~mm} \mathrm{SL}$ ) - MNHN 4611, 2 syntypes of Apistus trachinoides, 40.5-52.9 mm SL, Indonesia; MNHN 6643, 2 syntypes of A. trachinoides, 40.6-45.0 mm SL, Indonesia;
MNHN 6753, holotype of Prosopodasys bottae Sauvage 1878, $58.4 \mathrm{~mm}$ SL, Red Sea; USNM 52504, holotype of Prosopodasys gogorzae Jordan and Seale 1905, $21.2 \mathrm{~mm}$ SL, Philippines; KAUM-I. 24070, 42.6 mm SL, Thailand; KAUM-I. 24071, 56.9 mm SL, Thailand; KAUM-I. 24072, $58.8 \mathrm{~mm}$ SL, Thailand; KAUM-I. 44178, $46.1 \mathrm{~mm}$ SL, Thailand; KAUM-I. 44179, 47.4 mm SL, Thailand; KAUM-I. 52303, 41.9 mm SL, Malaysia; KAUM-I. 52321, 45.4 mm SL, Malaysia; KAUM-I. 57817, 49.7 mm SL, Thailand; KAUM-I. 77687, 52.9 mm SL, Vietnam; KAUM-I. 77688, 41.2 mm SL, Vietnam; MNHN 4610, $47.2 \mathrm{~mm}$ SL, Indonesia; URM-P 12442, 3, 45.6-51.8 mm SL, Thailand; USNM 87100, $44.7 \mathrm{~mm}$ SL, China; USNM 136326, $21.3 \mathrm{~mm}$ SL, Philippines; USNM 136327, 2, 33.3-36.1 mm SL, Malaysia; USNM 136398, 22.5 mm SL, Philippines; USNM 161427, 40.4 mm SL, Philippines; USNM 169192, 2, 37.0-45.4 mm SL, Malaysia; USNM 228900, $58.8 \mathrm{~mm}$ SL, Indonesia; USNM 403404, 42.8 mm SL, Philippines; USNM 408965, $75.8 \mathrm{~mm}$ SL, Philippines.

Publisher's Note Springer Nature remains neutral with regard to jurisdictional claims in published maps and institutional affiliations. 